\title{
TRANSITION IN PREFERRED ORIENTATION OF POLYCRYSTALLINE ICE FROM REPEATED RECRYSTALLIZATION
}

\author{
by
}

\author{
Huang MaOHUan
}

Lanzhou Institute of Glaciology and Geocryology, Academia Sinica, Lanzhou, China

\author{
M. OHтомo and G. WaKahama
}

Hokkaido University, Sapporo, Japan 060

\begin{abstract}
A repeated compression and annealing experiment was conducted using polycrystalline ice samples. The sample was compressed in steps of $10 \%$ at a constant low deformation rate at $272 \mathrm{~K}$, and annealed at the same temperature, thus allowing the sample to recrystallize. As a result, the ice gradually changed to a fabric pattern of the 4-maximum or 3-maximum type. The result indicates that repetitive recrystallization plays an important role in forming the preferred orientation of ice in glaciers.
\end{abstract}

\section{INTRODUCTION}

The flow of a glacier causes a transition in the distribution of orientation of crystallographic axes in individual grains of glacier ice (fabric). As a result, orientations are concentrated in space (preferred orientation). For example, one pattern has four maximums (the so-called diamond pattern with four concentrations seen in the distribution), which is well known as a characteristic pattern of glacier ice. Many investigators have tried to clarify the formation mechanisms of such patterns and to reproduce them experimentally (for a review see Paterson 1981), by providing some of the conditions of actual glacier flow. However, the mechanisms are still unclear; for example, the four-maximum pattern has not been reproduced experimentally. In new experiments using a polycrystalline ice we subjected a sample to repeated recrystallization, as occurs in actual glacier flow. replicating glacier conditions we reduced the strain to an amount sufficient to allow nucleation of the recrystallized grains, and following the actual glacier flow, the sample was finally annealed under low stress or in stress-free conditions.

\section{EXPERIMENTAL METHOD}

Polycrystalline ice samples used in this experiment were fine-grained and randomly oriented. First a rectangular solid sample, 10 x $10 \times 30 \mathrm{~cm}^{3}$ in dimensions, cut out from an ice block made from fine-grained snow saturated with distilled water. The block was uniaxially compressed at a constant rate of $0.005 \mathrm{~mm} / \mathrm{min}$ at $272 \mathrm{~K}$, until $10 \%$ strain was obtained. This was the lowest possible rate with our testing machine: the strain caused was nearly the minimum for recrystallization. We expected that these treatments would cause the sample to recrystallize gently, changing the texture without grain rotation. After compression the sample was annealed for 72 hours at the same temperature. Two types of annealing processes were adopted, one stress free, the other under a weak stress of $0.1 \mathrm{MPa}$, which is considered to be characteristic for glaciers. This annealing period was sufficient for the sample to complete primary recrystallization. A thin section was then sliced from the sample for measurements of data on fabric, misorientation angle between the c-axes of adjoining grains and grain size distribution. To prepare for the next process of compression and annealing the side faces of the deformed sample were restored to rectangular shape by carefully keeping both top faces parallel. The process was repeated 12 to 16 times in all.

\section{RESULTS}

Figure 1 shows transition of the fabric pattern of a sample annealed without stress. It transformed as follows. At the initial process a randomly oriented pattern changed, a girdle appearing around the compression axis (Figure 1a,b). Then the center of the girdle shifted from the compression axis (Figure 1c). Finally the girdle was separated into parts, resulting in formation of a fabric pattern of the four-maximum or three-maximum type (Figure 1d-f). Three samples which had been subjected to this experiment gave similar results, though the transition in the preferred orientation seemed to be accelerated in samples annealed under low stress. The frequency histogram of misorientation between adjoining grains, and grain size distribution, of the same sample after the 12 th annealing are shown in Figure $2 a$ and $2 b$ respectively. The misorientation angle between adjoining grains changed by degrees from random distribution at the beginning to a narrow range at the final process, and the mean diameter of grains decreased gradually.

\section{DISCUSSION}

Experimental results show clearly that the fabric pattern gradually transformed, reproducing the four- or three-maximum fabric pattern despite the stress field remaining unchanged. That is, the stable fabric pattern in the stress field, which has been reported by many investigators (eg Kamb 1972; Wilson and Russel-Head 1982), has not been shown in our experiment. This discrepancy, and the formation of the multi-maximum pattern, were obviously brought about from the repeated recrystallization process, the occurrence of which was confirmed in the thin sections.

The observed shift in the center of the girdle (Figure 1c-e) is hardly explained by sample rotation, because the sample was carefully set before it was compressed. We consider rather that it also originates from recrystallization. As shown in Figure la-e, the girdle pattern remains unchanged except for a decrease in diameter, which we interpret as follows. When some kind of preferred orientation is formed like this, there exist clusters of grains having small misorientation angles between them. Then, a recrystallized grain is unlikely to appear in a cluster (Fukuda unpublished). For this reason the decrease in diameter of the girdle may originate from rotation of each grain. Misorientations between individual maxima of the reproduced 4-maximum pattern and those between adjoining grains (Figure 2a) were close to those of samples from glaciers (Kizaki 1969; Matsuda and Wakahama 1978). Multi-maximum patterns were found in the field where recrystallization made the grain rather coarse (eg Kizaki 1969), in contrast to our results (Figure 2b). We explain 


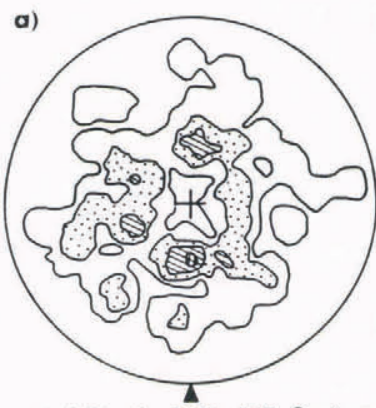

Total Strain $17 \% 102$ Grains

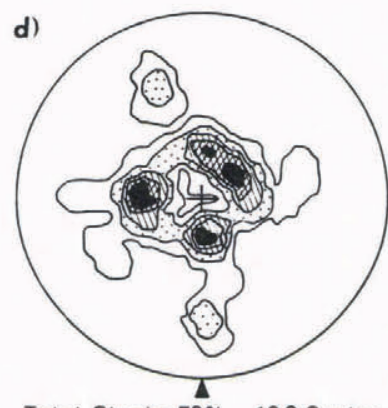

Total Strain $59 \% \quad 100$ Grains

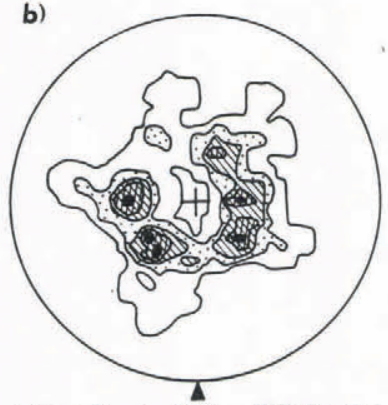

Total Strain $34 \% \quad 105$ Grains

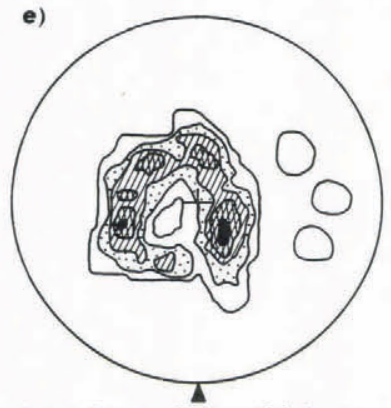

Total Strain $68 \% 100$ Grains

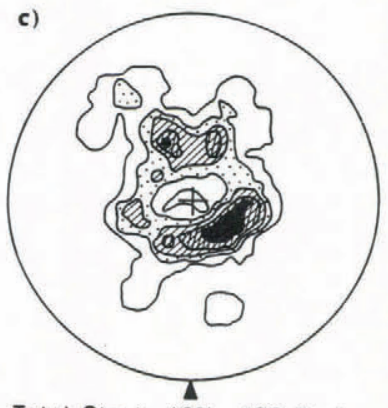

Total Strain $49 \% 100$ Grains

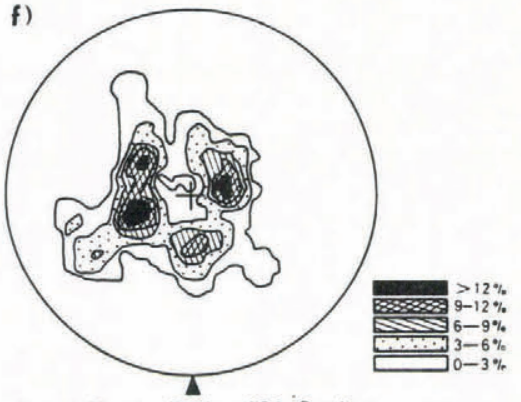

Total Strain $73 \% \quad 101$ Grains

Fig.1. Transition of the fabric pattern as a result of repeated recrystallization. The compression axis is the center of the projection circle. a, b, c, d, e, and f: after 2 nd, 4th, 6th, 8th, 10th, and 12th annealing, respectively.
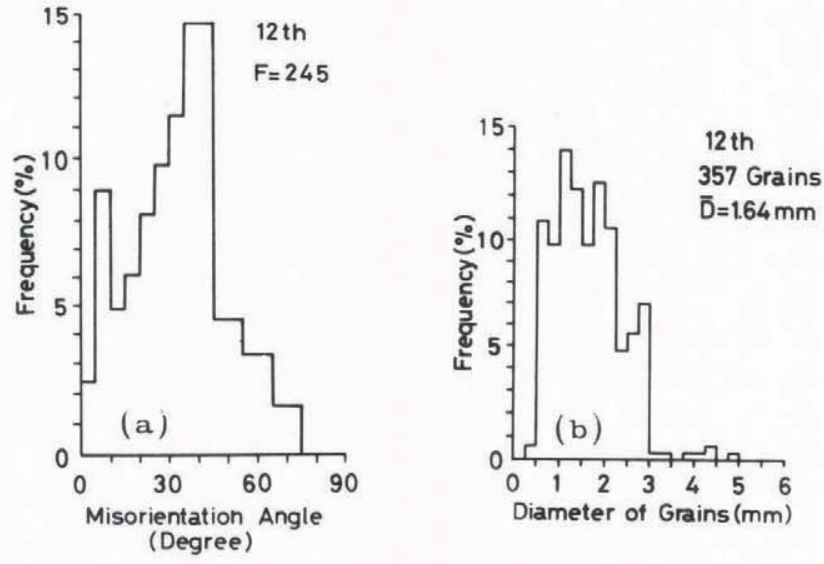

Fig.2. Frequency histogram after 12th annealing. (a) mis orientation angle between adjoining grains; (b) grain size distribution.

this as follows. With the repetition of compression the length of the sample was shortened, so that the strain rate increased. As a result, the nucleation rate speeded up, increasing the number of recrystallized grains.

Finally, as for the cause of the transition of the fabric pattern, we consider that the fabric pattern formed by recrystallization fairly depends upon the earlier distribution of c-axes, as Ohtomo and Wakahama (1982) showed that the orientation of the c-axis of the recrystallized grain depended upon that of the grain which was invaded by the recrystallized grain. Consequently, the role of the repetition of recrystallization process should be emphasized in the formation of the preferred orientation of ice in the glacier.

\section{ACKNOWLEDGEMENTS}

This work was conducted when Huang Maohuan stayed at the Institute of Low Temperature Science,
Hokkaido University as a research fellow. An acknowledgement is expressed to Professor A. Fukuda, Muroran Institute of Technology, for providing the unpublished data. The experimental data were processed at the Hokkaido University Computing Center.

\section{REFERENCES}

Kamb W B 1972 Experimental recrystallization of ice under stress. In Heard $\mathrm{H} \mathrm{C}$ and others (ed) Flow and fracture of rocks. Washington, DC, American Geophysical Union: 211-241. (Geophysical Monograph 16)

Kizaki K 1969 Ice-fabricstudy of the Mawson region, East Antarctica. Journal of Glaciology 8(53): 253-276

Matsuda M, Wakahama G 1978 Crystallographicstructure of polycrystalline ice. Journal of Glaciology 21(85): 606-620

Ohtomo M, Wakahama G 1982 [Crystallographic orientation of recrystallized grain grown in strained single crystal of ice]. Low Temperature Science A, 41: $1-11$

Paterson W S 1981 Physics of glacier. 2nd ed. Oxford, Pergamon Press

Wilson C J L, Russel-Head D S 1982 Steady-state preferred orientation of ice deformed in plane strain at $-1{ }^{\circ} \mathrm{C}$. Journal of Glaciology 28(98): 145-161 